\title{
Porcentagem de sacarose aparente e potencial produtivo de açúcar em cultivares de cana
}

\section{Percentage of apparent sucrose and productive potential of sugar in cane cultivars}

\author{
Hélio Francisco Da Silva Neto, Fernando Abackerli De Pauli*, Luiz Carlos Tasso Junior, \\ Marcos Omir Marques
}

Facultade de Ciencias Agrarias e Veterinarias Jaboticabal / SP - UNESP - Campus de Jaboticabal, SP. Via Prof. Paulo Donato Castellane acceso s/n. 14884-900 Jaboticabal.SP, Brasil.

Recibido 13 junio 2013; Aceptado 17 marzo 2014.

\begin{abstract}
Resumo
A porcentagem de sacarose aparente "POL" consiste em importante variável para a determinação da produção açucareira, sendo que o seu valor é distinto em diferentes cultivares, partes da planta e épocas de crescimento. O trabalho teve como objetivo comparar os valores da POL e estimar o potencial produtivo de açúcar do caldo extraído de nós e entrenós de cultivares de cana-de-açúcar ao longo do ano agrícola 2010/2011. Utilizou-se o delineamento experimental de blocos ao acaso, em esquema fatorial $3 \times 2 \times 6$, com quatro repetições. Os tratamentos principais foram os cultivares de cana-de-açúcar, os tratamentos secundários as duas partes do colmo de cana e o terceiro as épocas de análises distribuídas ao longo da safra. Os cultivares SP81-3250 e RB72454 apresentaram os maiores valores de POL. De modo contrário, o RB867515 obteve resultados menos satisfatórios, apresentando menor valor da POL durante todo o período estudado, e nas duas partes do colmo. O entrenó obteve maior POL, e apresentou maior potencial de produção de açúcar quando comparado à parte do colmo representada pelo nó. Os valores de POL e o potencial produtivo de açúcar, obtidos por ambas as partes do colmo, foram crescentes ao longo da safra, e coincidiram com a época de colheita recomendada para estes cultivares. Entretanto, foi neste período que ocorreu a maior diferença entre as partes do colmo, indicando a necessidade de um melhor aproveitamento do entrenó.
\end{abstract}

Palavras-chave: matéria-prima, entrenó, sacarose, variedades.

\begin{abstract}
The percentage of saccharose apparent "POL" is an important variable for the determination of sugar production, and its value differs among the cultivars, plant parts and growth seasons. The study aimed to compare values of POL and estimate productive potential of sugar in juice extracted from nodes and internodes of the sugarcane cultivars sugar during the 2010/2011 crop season. We used the experimental design of randomized blocks in factorial scheme $3 \times 2 \times 6$, with four replications. The main treatments were cultivars of sugarcane, the secondary treatments two parts of the stalks of sugarcane, and third the seasons of analysis distributed over the harvest. The cultivars RB72454 and SP81-3250 showed the highest values POL. Conversely, the RB867515 obtained less satisfactory results, showing lower value of POL throughout the study period, and the two parts of the stalk. The internode was greater than POL, and showed greater potential for sugar production when compared to the stalk portion represented by the node. The values of POL and the productive potential of sugar, obtained by both sides of the stalk were growing throughout the season, and coincided with the harvest season recommended for these cultivars. However, there was this period that the biggest difference between the parts of the stalk, indicating the need for a better use of the internode Keywords: raw material, internode, saccharose, varieties.
\end{abstract}

\section{Introdução}

A cana-de-açúcar é formada de raízes, flores e colmos (Segato, 2006). Os colmos, definidos como a parte acima do solo, são formados por nós e entrenós, os quais apresentam diferentes formatos e funções.

\footnotetext{
* Autor para correspondencia

E-mail: fernando_abackerli@hotmail.com (F. De Pauli).
} 
Sendo assim, a atividade metabólica da planta ocorre em sua maior parte nos nós, enquanto os entrenós têm como principal função o acúmulo da sacarose (Silva-Neto et al., 2010).

Dessa forma, a qualidade do caldo extraído dessas partes do colmo interfere, segundo a sua origem, na eficiência do processamento da cana (Silva-Neto et al., 2011). Dinardo-Miranda et al. (2008) revelaram ainda, que na parte do colmo representada pelo nó encontra-se um emaranhado de feixes vasculares, responsáveis pela distribuição de água e nutrientes para as folhas. Neste local é encontrado um maior teor de fibra $\mathrm{e}$ consequentemente menor teor de sacarose.

Neste sentido, em experimento realizado com cultivares de cana, Branco et al., (2010) observou maior teor de POL no entrenó $(13,97)$, quando comparado ao nó (12,08). Ripoli e Ripoli (2004) definiram que o teor ideal da POL deve ser maior que $14 \%$ para garantir uma boa eficiência do processo de produção de açúcar.

Dessa forma, comparando o potencial de produção de açúcar para os cultivares tardios de cana-de-açúcar. Feliciano (2009) observou aumento do potencial produtivo ao longo da safra, sendo constatado os maiores valores aos 17 meses e meio nas condições de cana-planta de ano e meio. A partir disso os valores apresentaram tendência de decréscimo.

Em relação às partes do colmo, o potencial produtivo de açúcar é diferente entre as partes do colmo. Homem et al. (2009), em experimento realizado apenas uma única analise da safra, obteve uma maior produção no entrenó (aproximadamente 20 quilogramas a mais do que no nó). Entretanto, os valores da POL o potencial de produção de açúcar podem se comportar de modo distinto ao longo da safra, e o conhecimento destes valores, para cada parte do colmo, pode auxiliar nas diversas etapas que compõe o processo de industrialização da cana.

Sendo assim, este trabalho teve como objetivo comparar os valore da POL e estimar o potencial produtivo de açúcar do caldo extraído de nós e entrenós de cultivares de cana-de-açúcar ao longo do ano agrícola 2010/2011.

\section{Material e métodos}

O experimento foi desenvolvido na Fazenda de Ensino, Pesquisa e Produção da Faculdade de Ciências Agrárias e Veterinárias/UNESP-Jaboticabal, SP. Sua altitude média é de 575 metros do nível do mar, e sua localização geográfica é definida como: latitude $21^{\circ} 15^{\prime} 22$ ' $\mathrm{S}$ e longitude $48^{\circ} 18^{\prime}$ '58' 'WG.

$\mathrm{O}$ experimento foi instalado em um Latossolo-Vermelho Escuro, eutrófico, A moderado, textura muito argilosa (EMBRAPA, 1999), relevo suave ondulado.

$\mathrm{O}$ delineamento experimental utilizado foi o de blocos ao acaso, em esquema fatorial $3 \times 2 \times 6$, com quatro repetições. Os tratamentos principais utilizados foram os três cultivares de cana-de-açúcar: RB867515, RB72454 e SP81-3250. Tais cultivares são classificados como de maturação do meio para o final de safra (Marques et al., 2008).

Os tratamentos secundários foram as duas partes do colmo de cana (nó e entrenó). O terceiro tratamento foram as épocas de análises distribuídas ao longo do ano agrícola 2010/2011: 0, 21, 49, 70, 105 e 153 dias após a análise inicial. A primeira análise (Época 0) foi realizada no dia 26/04/2010, aos 6 meses de idade, nas condições de cana soca de terceiro corte.

No campo o experimento era constituído por quatro blocos. Em cada bloco, os cultivares foram representados pelas parcelas experimentais, dispostas ao acaso. Cada parcela era composta por cinco linhas de cana com 12 metros de comprimento e espaçamento de 1,5 metros entre elas, totalizando $90 \mathrm{~m}^{2}$. Considerou-se como área útil para amostragem as três linhas centrais, descartando-se um metro de suas extremidades, resultando em $45 \mathrm{~m}^{2}$.

Foram coletados 15 colmos de cana por parcela, de cada bloco. Os colmos foram 
cortados em sequência na linha de plantio, de forma manual utilizando-se de facão. $O$ local de coleta foi escolhido aleatoriamente no momento da amostragem. Após o corte, os colmos foram despontados, despalhados, enfeixados, etiquetados e encaminhados ao Laboratório de Tecnologia do Açúcar e do Etanol da FCAV/UNESP.

No laboratório os colmos foram separados em nó e entrenó com o auxílio de uma serra elétrica modelo "Tico-tico". Depois de separadas, as partes foram encaminhadas para o desintegrador de cana. Foram pesados 500 gramas do material desintegrado, o qual foi encaminhado para a prensa hidráulica para extração do caldo. Após a filtragem do caldo em algodão hidrófilo foi realizado a análise tecnológica do caldo, sendo determinado o teor de açúcares redutores $(\mathrm{AR})$ e $\mathrm{o}$ teor de açúcares redutores totais (ART) por titulação, de acordo com o método de Lane e Eynon (1923).

Por meio de cálculo determinou-se os valores da POL\%cana. Os resultados obtidos foram submetidos à análise de variância. Quando houve significância, as médias foram comparadas pelo teste de Tukey, a 5\% de probabilidade. De posse destes resultados, foi estimada a produção de açúcar especial e VHP de acordo com as normas operacionais definidas pelo CONSECANA-SP (2006).

\section{Resultados e discussão}

Na tabela 1 são apresentados os valores da POL obtidos pelos cultivares e partes do colmo ao longo do tempo analisado. Os cultivares SP81-3250 e RB72454 obtiveram os maiores valores, enquanto $o$ cultivar RB867515 apresentou o menor $(10,86)$. Para todos os exemplares os valores encontram-se abaixo do valor recomendado por Ripoli e Ripoli (2004), que preconiza uma POL de $14 \%$.

Em relação às partes do colmo, o entrenó obteve maior valor de POL $(12,80 \%)$ quando comparado ao nó $(9,99 \%)$. Uma possível explicação para este resultado seria de que no entrenó é acumulada a maior parte da sacarose (Silva-Neto et al., 2010). Na média observou-se aumento dos valores da POL ao longo da safra, e com isso as épocas finais apresentaram os maiores valores.

\section{Tabela 1}

Valores médio de POL (porcentagem de sacarose aparente na cana) obtidos pelas diferentes partes do colmo dos cultivares de cana-de-açúcar, além da análise estatística.

\begin{tabular}{lc}
\hline Causas de variação & POL \\
\hline Cultivares (C) & $10,86 \mathrm{~b}$ \\
RB867515 & $11,54 \mathrm{a}$ \\
RB72454 & $11,76 \mathrm{a}$ \\
SP81-3250 & $12,80 \mathrm{a}$ \\
\hline Partes (P) & $9,99 \mathrm{~b}$ \\
Entrenó & \\
Nó & $8,11 \mathrm{e}$ \\
\hline Épocas (E) & $9,28 \mathrm{~d}$ \\
0 & $10,97 \mathrm{c}$ \\
21 & $12,49 \mathrm{~b}$ \\
49 & $14,76 \mathrm{a}$ \\
70 & $12,72 \mathrm{~b}$ \\
105 & \\
153 & $2,90^{\mathrm{NS}}$ \\
\hline Estatística-Teste F & $76,43^{* *}$ \\
Blocos & $2980,75^{* *}$ \\
Cultivares (C) & $512,51^{* *}$ \\
Partes(P) & $12,69^{* *}$ \\
Épocas (E) & $4,14^{* *}$ \\
Interação (C) x (P) & $29,94^{* *}$ \\
Interação (C) x (E) & $1,94^{\mathrm{NS}}$ \\
Interação (P) x (E) & \\
Interação (C) x (P) x (E) & \\
\hline Coeficiente de Variação & 3,26 \\
(CV\%) & 2,72 \\
Cultivares (C) & 4,63 \\
Partes (P) & Épocas (E)
\end{tabular}

Médias seguidas de letras iguais, na coluna, em cada atributo, não diferem entre si pelo teste de Tukey, ao nível de $5 \%$ de probabilidade. NS e ** Não significativo e significativo ao nível de $1 \%$ de probabilidade (Teste F), respectivamente.

Na tabela 2 é apresentada a interação obtida entre cultivares e partes do colmo. $\mathrm{Na}$ comparação entre os cultivares para uma mesma parte do colmo pode-se observar que o cultivar SP81-3250 apresentou os maiores valores da POL. De modo inverso, o cultivar RB867515 obteve 
os menores valores na comparação entre os cultivares para o entrenó e também para nó.

Na comparação entre as partes do colmo os três cultivares apresentaram os maiores valores de ART no entrenó. Ressalta-se ainda, que o cultivar SP81-3250 apresentou a maior diferença entre as partes estudadas, sendo que o nó obteve $23,74 \%$ a menos de POL em relação ao entrenó. Resultados semelhantes foram obtidos por Branco et al. (2010) que também constatou a superioridade do entrenó quando comparado ao nó, 13,97 e $12,08 \%$, respectivamente. Estes resultados podem ter ocorrido pelo fato de que nos entrenós os feixes encontram-se dispostos de forma paralela entre si, enquanto na região nodal os feixes apresentam-se emaranhados uns aos outros (DinardoMiranda et al., 2008), ocupando uma maior proporção dos tecidos que compõem o colmo.

$\mathrm{Na}$ tabela 3 é apresentado o efeito da interação entre cultivares e épocas de análise. Todos os cultivares estudados apresentaram aumento dos teores de POL ao longo do período analisado. $\mathrm{O}$ maior valor foi encontrado na época 105, aproximadamente aos 10 meses de idade do canavial. Feliciano (2009) também obteve aumento da qualidade do caldo ao longo da safra, porém seu maior valor ocorreu aos 17 meses. Entretanto, é importante ressaltar que o trabalho citado foi realizado nas condições de cana-planta de ano e meio.

A tabela 4 apresenta o desdobramento entre as épocas e as partes do colmo. Em todas as épocas o entrenó foi superior ao nó, sendo que a maior diferença ocorreu no período de maior acúmulo de açúcar (Época 105) e na época recomendada de colheita destes cultivares (Marques et al., 2008). Dessa forma, fica evidente a melhor qualidade caldo no entrenó, mesmo em épocas iniciais de cultivo da cana até o ponto de colheita.

\section{Tabela 2}

Efeito da Interação obtida entre cultivares (C) e partes do colmo (P), para os teores de POL\%cana.

\begin{tabular}{lcrr}
\hline Cultivares & Entrenó & \multicolumn{1}{c}{ Nó } & \multicolumn{1}{c}{ Teste $\mathrm{F}$} \\
\hline RB867515 & $12,23 \mathrm{Ca}$ & $9,50 \mathrm{Bb}$ & $929,06^{* *}$ \\
SP81-3250 & $13,35 \mathrm{Aa}$ & $10,18 \mathrm{Ab}$ & $1259,38^{* *}$ \\
RB72454 & $12,82 \mathrm{Ba}$ & $10,27 \mathrm{Ab}$ & $817,69^{* *}$ \\
\hline Teste F & $64,85^{* *}$ & $35,65^{* *}$ &
\end{tabular}

Letras maiúsculas comparação na vertical. Letras minúsculas comparação na horizontal. Médias seguidas de letras iguais não diferem entre si pelo teste de Tukey, ao nível de 5\% de probabilidade. ** Significativo ao nível de $1 \%$ de probabilidade (Teste F).

\section{Tabela 3}

Desdobramento da interação entre cultivares (C) e épocas de análise (E), para os teores de POL\%cana.

\begin{tabular}{llcccccr}
\hline \multirow{2}{*}{ Cultivares } & \multicolumn{7}{c}{ Época } \\
\cline { 2 - 8 } & \multicolumn{1}{c}{0} & 21 & \multicolumn{1}{c}{49} & 70 & 105 & 153 & Teste $\mathrm{F}$ \\
\hline RB867515 & $7,77 \mathrm{Be}$ & $8,90 \mathrm{Bd}$ & $10,70 \mathrm{Bc}$ & $11,82 \mathrm{Bb}$ & $13,67 \mathrm{Ca}$ & $12,32 \mathrm{Bb}$ & $140,29 * *$ \\
SP81-3250 & $8,06 \mathrm{Abe}$ & $9,81 \mathrm{Ad}$ & $10,92 \mathrm{Abc}$ & $13,11 \mathrm{Ab}$ & $15,68 \mathrm{Aa}$ & $13,00 \mathrm{Ab}$ & $212,57 * *$ \\
RB72454 & $8,51 \mathrm{Ad}$ & $9,13 \mathrm{Bd}$ & $11,31 \mathrm{Ac}$ & $12,53 \mathrm{Ab}$ & $14,92 \mathrm{Ba}$ & $12,83 \mathrm{Abb}$ & $167,91 * *$ \\
\hline Teste F & $4,33^{* *}$ & $7,03 * *$ & $2,95^{\mathrm{NS}}$ & $13,40 * *$ & $32,38^{* *}$ & $3,80 * *$ \\
\hline
\end{tabular}

Letras maiúsculas comparação na vertical. Letras minúsculas comparação na horizontal. Médias seguidas de letras iguais não diferem entre si pelo teste de Tukey, ao nível de 5\% de probabilidade. ** Significativo ao nível de $1 \%$ de probabilidade. ${ }^{\mathrm{NS}}$ Não significativo. 


\section{Tabela 4}

Efeito da interação entre partes do colmo $(\mathrm{P})$ e épocas de análise (E), para os valores de POL\% cana.

\begin{tabular}{lccc}
\hline Épocas & Entrenó & Nó & Teste $\mathrm{F}$ \\
\hline 0 & $8,75 \mathrm{Ae}$ & $7,47 \mathrm{Be}$ & $39,76^{* *}$ \\
21 & $10,36 \mathrm{Ad}$ & $8,20 \mathrm{Bd}$ & $113,61^{* *}$ \\
49 & $12,10 \mathrm{Ac}$ & $9,85 \mathrm{Bc}$ & $123,48^{* *}$ \\
70 & $14,59 \mathrm{Ab}$ & $10,38 \mathrm{Bc}$ & $428,67^{* *}$ \\
105 & $16,86 \mathrm{Aa}$ & $12,66 \mathrm{Ba}$ & $427,02^{* *}$ \\
153 & $14,12 \mathrm{Ab}$ & $11,32 \mathrm{Bb}$ & $189,95^{* *}$ \\
\hline Teste $\mathrm{F}$ & $381,69^{* *}$ & $160,75^{* *}$ & \\
\hline
\end{tabular}

Letras maiúsculas comparação na horizontal. Letras minúsculas comparação na vertical. Médias seguidas de letras iguais não diferem entre si pelo teste de Tukey, ao nível de 5\% de probabilidade. ** Significativo ao nível de $1 \%$ de probabilidade.

Nas figuras 1 e 2 são expressos os valores médios da estimativa do potencial produtivo dos nós e entrenós da cana-deaçúcar para o açúcar especial e VHP, respectivamente. Para ambas as partes houve aumento do potencial de produção até a época 105, seguido de uma diminuição na última análise. Porém, desde o início o entrenó foi superior ao nó, sendo que nas épocas inicias a diferença entre eles foi de aproximadamente 10 quilogramas de açúcar por tonelada. De modo semelhante, esta diferença entre as partes também foi comprovada por Homem et al. (2009), em experimento realizado apenas em uma única analise da safra, obteve uma maior produção no entrenó de aproximadamente 20 quilogramas a mais do que no nó.

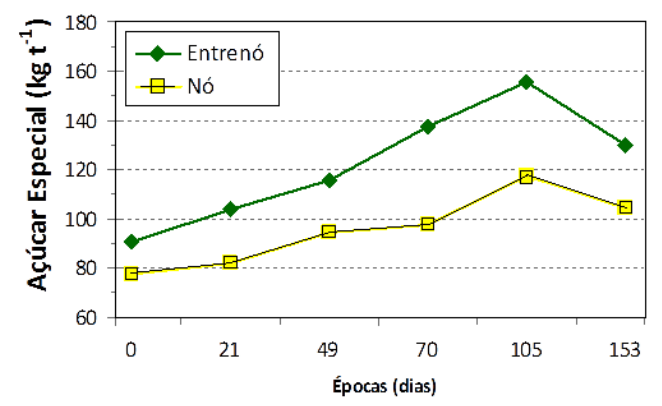

Figura 1. Potencial produtivo de açúcar especial (expresso em kilogramas por tonelada) obtido pelas partes do colmo de cana-de-açúcar ao longo do periodo analisado.
A maior diferença entre as partes do colmo ocorreu nas épocas finais, sendo que o entrenó, na época 105, apresentou 40 quilogramas de açúcar a mais por tonelada quando comparado ao nó. É importante ressaltar, para o ciclo de maturação destes cultivares, a colheita seria realizada justamente no período de maior diferença na produção de açúcar entre as partes do colmo.

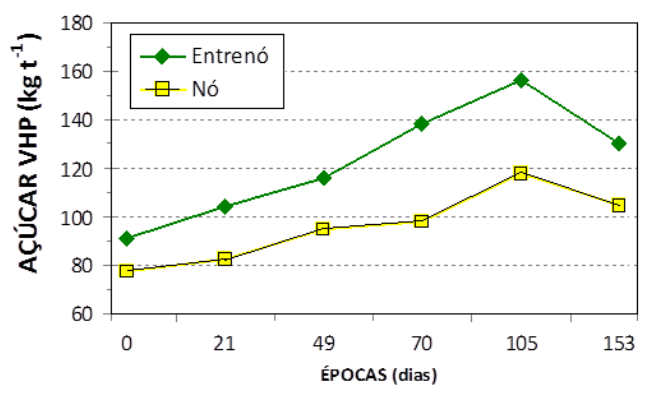

Figura 2. Potencial produtivo de açúcar VHP (expresso em kilogramas por tonelada) obtido pelas partes do colmo de cana-de-açúcar ao longo do periodo analisado.

\section{Conclusões}

Os cultivares SP81-3250 e RB72 454 apresentaram os maiores valores de POL, de modo contrário, o cultivar RB867515 obteve resultados menos satisfatórios, apresentando menor valor de POL durante todo o período estudado e nas duas partes do colmo. 
O entrenó obteve maior POL, e apresentou maior potencial de produção de açúcar quando comparado a parte do colmo representada pelo nó.

Os valores de POL e o potencial produtivo de açúcar, obtidos por ambas as partes do colmo, foram crescentes ao longo da safra, e coincidiram com a época de colheita recomendada para estes cultivares. Entretanto, foi neste período que ocorreu a maior diferença entre as partes do colmo, indicando a necessidade de um melhor aproveitamento do entrenó.

\section{Referências bibliográficas}

Branco, R.V.; Silva-Neto, H. F.; Silva J. D.R.; Marques, M.O. 2010. Variáveis tecnológicas de nós e entrenós de cana-de-açúcar (cultivares IACSP94-2101, IACSP93-3046 e IACSP94-4004). p. 1-4. In: SICUSP, 18. Piracicaba/SP Brasil.

CONSECANA-SP - Conselho dos produtores de cana-deaçúcar, açúcar e álcool do Estado de São Paulo. 2006. Manual de Instruções. Piracicaba: CONSECANA. 112 p.

Dinardo-Miranda, L.L.; Vasconcelos, A.C.M De; Landell, M.G.A. 2008. Cana-de áçúcar. Campinas: Instituto Agronômico. 882p.

EMBRAPA - Empresa Brasileira de Pesquisa Agropecuária. 1999. Centro Nacional de Pesquisa de Solos. Sistema brasileiro de classificação de solos. Embrapa. 412p.
Feliciano, R.F. 2009. Estudo comparativo entre cultivares de cana-de-açúcar em relação a conceitos de produção e margens de contribuição. Trabalho de Graduação (Engenharia Agronômica). FCAV/UNESP. Jaboticabal/SP, Brasil 104p.

Homem, R.F.M.; Silva-Neto, H.F.; Tasso-Júnior, L.C.; Marques, M.O. 2009. Açúcar total recuperável e produtividade de sacarose por cultivares de cana-deaçúcar. p.1-4. In: Congresso de Iniciação Cientifica da UNESP, 21. Jaboticabal/SP. Brasil. Editora, Jaboticabal/SP, Brasil.

Lane, H.; Eynon, L. 1923. Determination of reducing sugar by means of Fehling's solution with methylene blue as internal indicator. Journal of the Society of Chemistry Industry 42(1):.32-37.

Marques M.O.; Mutton, M.A.; Nogueira, T.A.R.; TassoJúnior, L.C.; Nogueira, G.A.; Bernardi, J.H. 2008. Tecnologias na Agroindústria Canavieira. FCAV. Jaboticabal/SP. 319p.

Ripoli, T.C.C.; Ripoli, M.L.C. 2004. Biomassa de cana-deaçúcar: colheita, energia e ambiente. Barros \& Marques Ed. Eletrônica. Piracicaba. 302 p.

Segato, S.V. 2006. Atualização em produção de cana-deaçúcar. Piracicaba: CP., 415p.

Silva-Neto, H.F.; Tasso-Junior, L.C.; Silva, J.D.R.; Marques, D.; Marques, M.O. 2010. Nós e entrenós de cana de açúcar armazenados por 168 horas. p.1-4. In: Congresso Latinoamericano y del Caribe de Ingenieria Agricola, 9 (CLIA) - Congresso Brasileiro De Engenharia Agricola, 39 (CONBEA). Vitoria/ES. Brasil. 25 a 29 de julho 2010. Vitória/ES, Brasil.

Silva-Neto, H.F.; Marques, M.O.; Da Silva, J.D.R.; TassoJúnior, L.C. 2011. Limitações e avanços tecnológicos na indústria da cana-de-açúcar. Disponível em: <www.diadecampo.com.br.> Acesso em: 28 de março de 2013. 